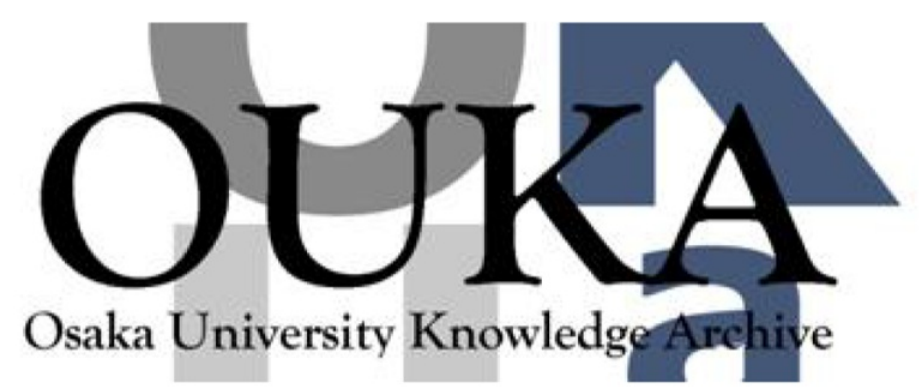

\begin{tabular}{|c|l|}
\hline Title & $\begin{array}{l}\text { Dual ring laser emission of conducting polymers } \\
\text { in microcapillary structures }\end{array}$ \\
\hline Author(s) & Yoshida, Y; Nishimura, T; Fuji i, A et al. \\
\hline Citation & $\begin{array}{l}\text { Applied Physics Letters. 86(14) p. 1414903- } \\
\text { p. 141903 }\end{array}$ \\
\hline Issue Date & $2005-03-30$ \\
\hline oaire:version VoR \\
\hline URL & https://hdl. handle.net/11094/75656 \\
\hline rights & \\
\hline Note & \\
\hline
\end{tabular}

Osaka University Knowledge Archive : OUKA

https://ir. Library. osaka-u. ac. jp/

Osaka University 


\section{Dual ring laser emission of conducting polymers in microcapillary structures}

Cite as: Appl. Phys. Lett. 86, 141903 (2005); https://doi.org/10.1063/1.1899229

Submitted: 16 December 2004 . Accepted: 22 February 2005 . Published Online: 30 March 2005

Y. Yoshida, T. Nishimura, A. Fujii, M. Ozaki, and K. Yoshino

\section{ARTICLES YOU MAY BE INTERESTED IN}

Ultralow sensing limit in optofluidic micro-bottle resonator biosensor by self-referenced differential-mode detection scheme

Applied Physics Letters 104, 033703 (2014); https://doi.org/10.1063/1.4861596

Hemispherical resonators with embedded nanocrystal quantum rod emitters

Applied Physics Letters 97, 211101 (2010); https://doi.org/10.1063/1.3517566

High-Q conical polymeric microcavities

Applied Physics Letters 96, 013303 (2010); https://doi.org/10.1063/1.3280044

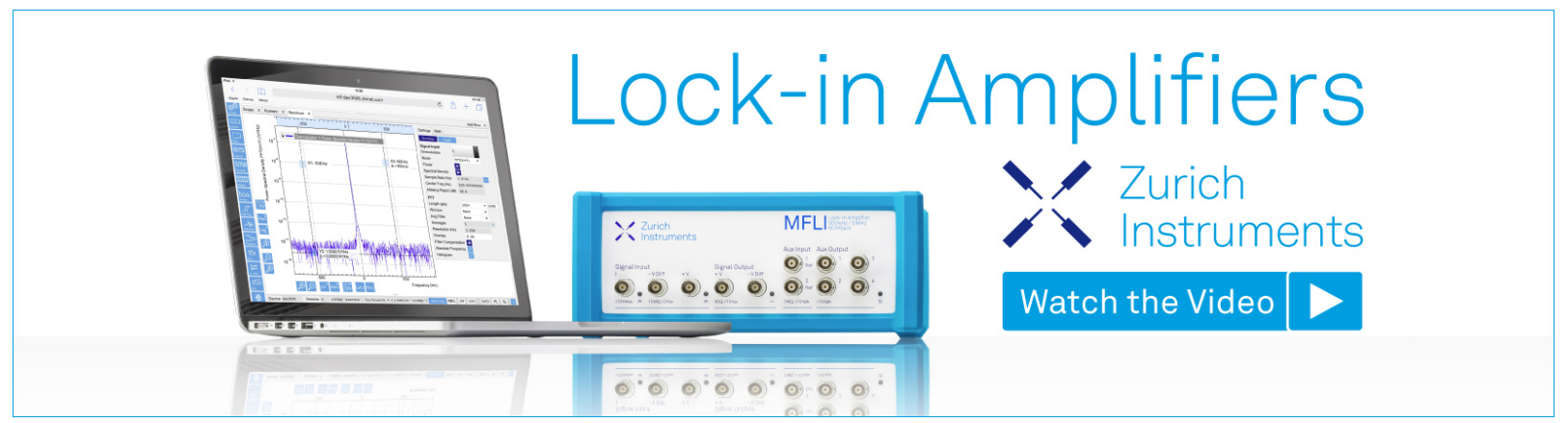




\title{
Dual ring laser emission of conducting polymers in microcapillary structures
}

\author{
Y. Yoshida, T. Nishimura, A. Fujii, M. Ozaki, and K. Yoshino \\ Department of Electronic Engineering, Graduate School of Engineering, Osaka University, \\ 2-1 Yamada-oka, Suita, Osaka 565-0871, Japan
}

(Received 16 December 2004; accepted 22 February 2005; published online 30 March 2005)

\begin{abstract}
We have demonstrated photopumped multimode laser emission from cylindrical microcavities of conducting polymer thin films, which were formed by deposition on the inside surface of the glass microcapillary with capillary action. The laser emission was characterized by narrow emission lines and a well-defined excitation threshold. We also fabricated dual ring cavities on inner and outer surfaces of microcapillaries, and laser emission from both sides of capillaries was observed by photopumping with one excitation source. Fabricating two cylindrical microcavities on inner and outer surfaces of the microcapillary by utilizing a different sort of conducting polymers, dual laser emissions, blue and red in color, were obtained concurrently. (C) 2005 American Institute of Physics. [DOI: 10.1063/1.1899229]
\end{abstract}

Rapid progress has been made in the synthesis and the investigation of conjugated polymers over the last two decades. Their intrinsic emissive properties make them promising materials for light-emitting devices, ${ }^{1-3}$ and solid-state lasers. Among various conducting polymers, alkyl- or alkoxy-substituted polymers, such as poly(3-alkylthiophene), ${ }^{2}$ poly(9,9-dialkylfluorene), ${ }^{3,4}$ and poly (2,5-dialkoxy-p-phenylene vinylene $)^{5,6}$ are the most attractive materials, because of their fusibility at relatively low temperatures, solubility in common solvents, and their high luminescent quantum efficiency. Furthermore, stimulated emission has been observed in photopumped thin films, resulting in spectral narrowing of the emission from conducting polymers. 7,8

Polymer lasers with microcavity structures have attracted much attention because of simple preparation procedure, low cost, and low laser threshold. Among various types of microcavities, we have demonstrated microring and microdisk lasers previously. ${ }^{8-10}$ In microring laser structures, the conducting polymer film is formed on the surface of a glass fiber.

In this letter, we demonstrate multimode laser emission from the conducting polymer film in microcapillary geometry.

Two kinds of conducting polymers, poly(2-methoxy-5dodecyloxy- $p$-phenylenevinylene) (MDDOPPV), and poly(9,9-dioctylfluorene) (PDAF8), were used in the present work. These polymers have the high photoluminescence (PL) efficiencies; therefore, they are suitable for emission devices and laser application. $^{3-6}$

The microcapillaries with diameters of around $30-200 \mu \mathrm{m}$ could be obtained by drawing glass tubes in a high-temperature flame. To form a microcavity structure of conducting polymer, the microcapillaries were dipped into a chloroform solution of these conducting polymers, and polymer neat films were deposited on the inside surface of the microcapillaries with capillary action spontaneously.

For the PL measurements at high excitation intensities, we used a $\mathrm{Nd}$ : yttrium aluminum garnet (YAG) regenerative laser amplifier producing 100 ps pulses with a repetition rate of $1 \mathrm{kHz}$. This laser light was frequency doubled (532 nm) and tripled $(355 \mathrm{~nm})$. The pump laser beam was focused by a round lens onto the microcapillaries with polymer films, and the emission from the microcapillaries was detected by a spectrometer and a charge-coupled device array with spectral resolution of approximately $0.2 \mathrm{~nm}$. The samples were held in a vacuum cell to avoid degradation by irradiation and oxidation.

Figure 1(a) shows the emission spectrum of the MDDOPPV film with a microring structure formed on the inside surface of the microcapillary at the pumping intensity of about $10 \mathrm{~nJ} /$ pulse, and the inset shows the molecular structure of MDDOPPV. The diameter of the microcavity D was approximately $150 \mu \mathrm{m}$. The inset of Fig. 1(b) shows a schematic image of a cross-sectional view of the polymer capillary structures. The sharp emission lines as shown in Fig. 1(a) appear only above a threshold excitation intensity $I_{0}$, which is determined to be approximately $2 \mathrm{~nJ} /$ pulse from the results in Fig. 1(b). Since the sharp lines were not confirmed at low pumping intensity, it is considered that the origin of the sharp lines is not a quantum electrodynamic enhancement of PL in a microcavity. ${ }^{11}$ These sharp lines, therefore, can be attributed to laser modes associated with the cylindrical microcavity consisting of the thin polymer film. The linewidth of the narrow laser lines was about $0.2 \mathrm{~nm}$, which is limited by the spectral resolution. The spectral spacing $(\Delta \lambda)$ between the laser lines was described as ${ }^{8-10}$

$$
\Delta \lambda=\lambda^{2} / \pi \mathrm{D} n_{\mathrm{eff}}
$$

From this equation, we calculated the effective refraction index to be $n_{\text {eff }}=1.67$ with $\Delta \lambda=0.48 \mathrm{~nm}$, which approximately coincided with that of bulk polymers.

These microcapillaries should have an excellent advantage; that is, inner emissive layers are protected just by closing the glass tube from ambient gases.

To utilize both the inner and outer surfaces of microcapillaries, the MDDOPPV film was also fabricated onto the outside of the capillary by the conventional method. ${ }^{8}$ Figure 2 shows the emission spectrum of the dual-microcavity structure of MDDOPPV at the excitation of $4.4 \mathrm{~nJ} /$ pulse. The spectrum contains different $\Delta \lambda$ values because of two microcavities on the inner and outer surfaces. One type of 

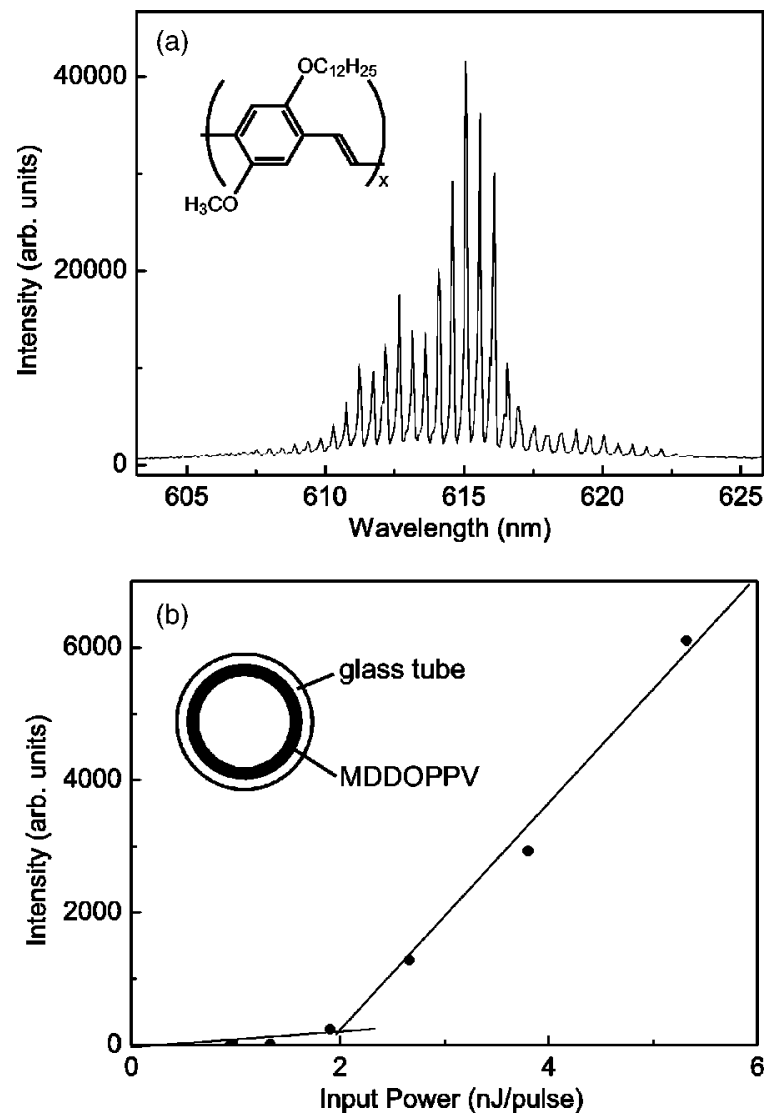

FIG. 1. (a) Lasing spectra of MDDOPPV film in the microring structure formed on the inside surface of the microcapillary. The inset shows the molecular structure of MDDOPPV. (b) The excitation intensity dependence of emission intensity from MDDOPPV microcapillary. The inset shows schematically the microcapillary structure.

sharp lines, with a line separation $\Delta \lambda_{1}$ of $1.14 \mathrm{~nm}$, corresponds to the laser from the inner cavity. The other set of lines with $\Delta \lambda_{2}$ of $1.03 \mathrm{~nm}$ originates from the outer cavity. The diameters of the inner and outer cavities were 63 and $79 \mu \mathrm{m}$, respectively. From Eq. (1), the effective refraction indices were calculated to be 1.59-1.81. Such an overlapping laser emission could be observed at the higher excitation than $1.4 \mathrm{~nJ} /$ pulse, which is the threshold energy estimated from the result of the inset of Fig. 2.

Using the microcapillary configuration, we also produced a microcavity with PDAF8 on the outside surface of a

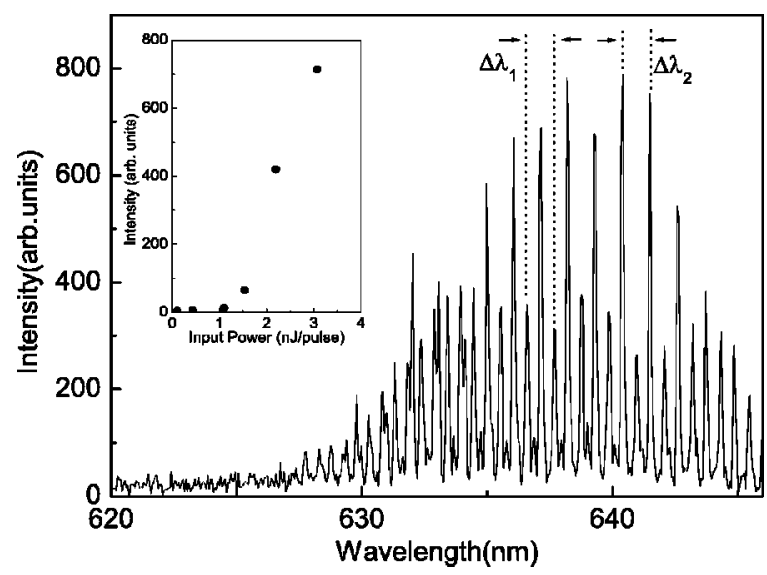

FIG. 2. Lasing spectra of MDDOPPV films with microring structure formed on both the outside and the inside surfaces of the microcapillary. In the inset is shown the emission intensity dependence on the excitation energy/pulse.
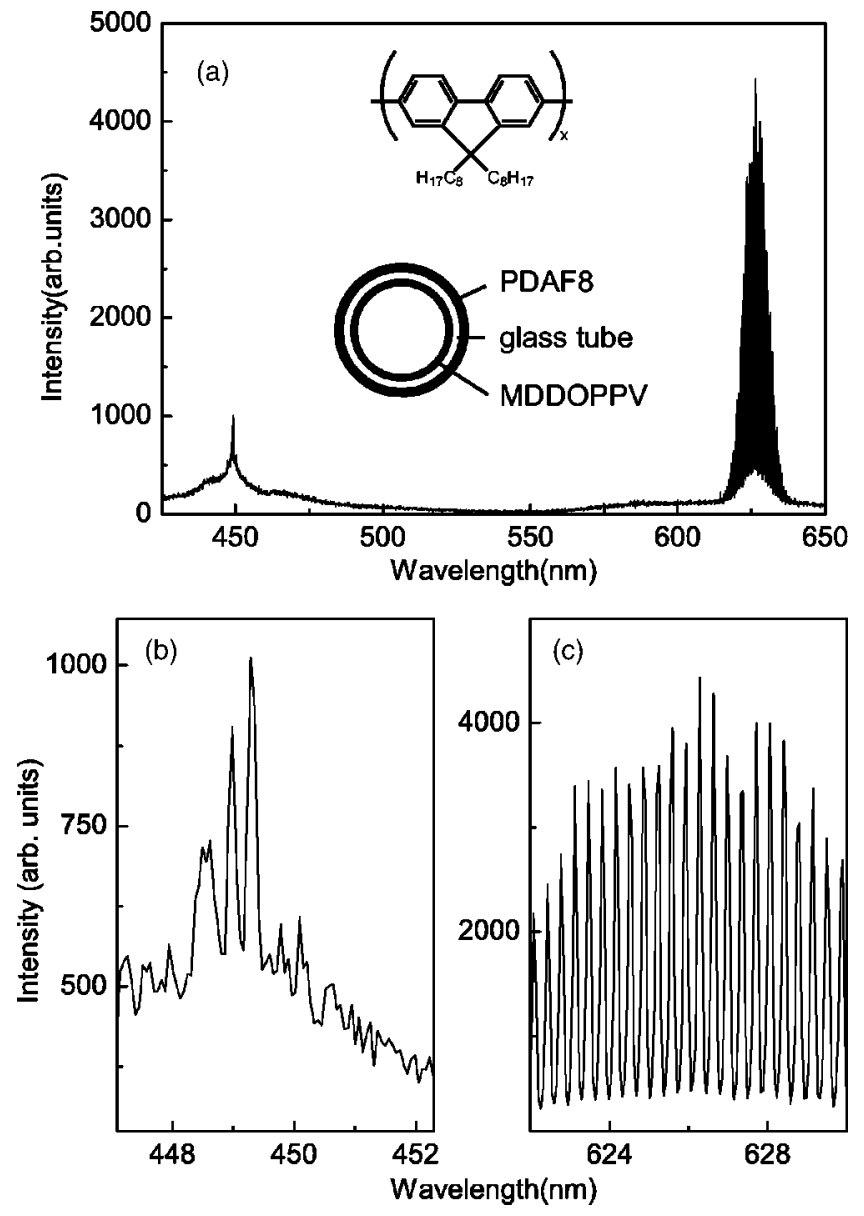

FIG. 3. (a) Lasing spectra of PDAF8 film at the outside of the microcapillaries and MDDOPPV film in the inside. Insets show the molecular structure of conducting polymer PDAF8 and schematically the two-microcavity structures of a microcapillary. The emission spectra under higher magnification in blue region (b) and in red region (c).

microcapillary, and that with MDDOPPV on the inside surface of the microcapillary. The molecular structure of PDAF8 and the schematic image of the double microrings consisting of MDDOPPV and PDAF8 are shown in the inset of Fig. 3(a). The blue and red laser emissions were observed from the microcapillary, as shown in Fig. 3(a). Spectral features of lasing in the blue and red regions are shown in Figs. 3(b) and 3(c), with higher magnification, respectively. These spectra indicate the resonant cavity modes and concurrent laser emissions with blue and red colors.

Although some ring-shaped cavities have already been known as laser cavities, such structures of two different cavities with one excitation source have not been reported so far. It should be emphasized that these cavities could be fabricated by the simple method. It could be a candidate of lowcost multicolor polymer laser devices.

In summary, we demonstrated multimode lasing from the microring structure of conducting polymers formed on the inside surface of microcapillaries by capillary action. The dual wavelength lasing, which was blue and red lights in color, was realized with microring structures of heterologous conducting polymer films formed on the inside and outside surfaces of the microcapillaries.

This work was partly supported by the Industrial Technology research Grant Program in '04 from the New Energy and Industrial Technology Development Organization 
(NEDO) of Japan, and grant-in-aid for JSPS fellows of the Ministry of Education, Culture, Sports, Science, and Technology.

${ }^{1}$ J. H. Burroughes, D. D. C. Bradley, A. R. Brown, R. N. Marks, K. Mackay, R. H. Friend, P. L. Burns, and A. B. Holmes, Nature (London) 347, 539 (1990)

${ }^{2}$ Y. Ohmori, M. Uchida, K. Muro, and K. Yoshino, Jpn. J. Appl. Phys., Part 2 30, L1938 (1991).

${ }^{3}$ Y. Ohmori, M. Uchida, K. Muro, and K. Yoshino, Jpn. J. Appl. Phys., Part 2 30, L1941 (1991).

${ }^{4}$ M. Fukuda, K. Sawada, and K. Yoshino, Jpn. J. Appl. Phys., Part 2 28, L1433 (1989).

${ }^{5}$ M. Hamaguchi and K. Yoshino, Jpn. J. Appl. Phys., Part 2 33, L1478
(1994).

${ }^{6}$ M. Hamaguchi and K. Yoshino, Jpn. J. Appl. Phys., Part 2 34, L712 (1995).

${ }^{7}$ Y. Yoshida, Y. Nishihara, R. Ootake, A. Fujii, M. Ozaki, K. Yoshino, H. K. Kim, N. S. Baek, and S. K. Coi, J. Appl. Phys. 90, 6061 (2001).

${ }^{8}$ Y. Yoshida, Y. Nishihara, A. Fujii, M. Ozaki, K. Yoshino, H. K. Kim, N. S. Baek, and S. K. Choi, J. Appl. Phys. 95, 4193 (2004).

${ }^{9}$ S. V. Frolov, A. Fujii, D. Chinn, M. Hirohata, R. Hidayat, M. Taraguchi, T. Masuda, K. Yoshino, and Z. V. Vardeny, Adv. Mater. (Weinheim, Ger.) 10, 869 (1998).

${ }^{10}$ S. V. Frolov, M. Shkunov, Z. V. Vardeny, and K. Yoshino, Phys. Rev. B 56, R4363 (1997).

${ }^{11}$ A. J. Campillo, J. D. Eversole, and H. B. Lin, Phys. Rev. Lett. 67, 437 (1991). 